\title{
Competition between Relaxation Kinetics and Heat Transfer in the Dynamics of Phase Transition Fronts
}

\author{
A. Gordon, S. Dorfman* and D. Fuks** \\ Department of Mathematics and Physics, Haifa University at Oranim, 36006 Tivon, Israel \\ * Department of Physics, Technion - Israel Institute of Technology, 32000 Haifa, Israel \\ ** Materials Engineering Department, Ben-Gurion University of the Negev, P.O. Box 653, \\ 84105 Beer Sheva, Israel
}

\begin{abstract}
The velocity of the interphase boundary motion caused by latent heat transfer at ferroelectric phase transitions is calculated. The criterion for separating of two mechanisms for the front motion - the latent heat transfer and the relaxation of the order parameter - is obtained for ferroelectrics. Electric field dynamics of interphase boundaries have been studied. The expression for the front velocity of the threshold field type is derived.
\end{abstract}

The transition from a metastable phase to a thermodynamically stable phase takes place via fluctuations leading to the formation of nuclei of the new phase (heterophase fluctuations) [1]. In order to describe systems which undergo a symmetry-breaking first-order phase transition, the nucleation and growth processes must be accounted for. The growth is associated with the propagation of interphase boundaries separating the high-temperature parent phase and the low-temperature product phase. In solid diffusionless transformations the growth may be slow enough for the observation by polarization microscope technique. In particular, in ferroelectrics sharp interphase boundaries can be observed [2-12]. Crystals with sharp interfaces most often have kinetically controlled rather than diffusion-limited growth. Usually the interphase dynamics are governed entirely by the time evolution of the order parameter [13-20], and the temperature can be considered to be a constant. Thus the heat is assumed to be removed rapidly enough that no temperature change occurs as the latent heat of the phase transition appears at the interphase boundary. However as the interface moves it acts as a heat source with a strength proportional to the latent heat and the forward rate of motion, giving rise to a jump in the thermal gradient. The heat generated during the interphase boundary motion can accelerate the interface which, in turn, increases the heat production rate. A system involved in this avalanche-like process can be stabilized by the heat removal via the heat conductivity and the heat exchange with the thermal bath. This problem is less important in metallic systems. If one deals with substances which conduct heat very well, the temperature may be treated as a constant and so no kinetic equation additional to one for the order parameter is necessary. But for materials which do not conduct heat so well, we may need a second equation to determine the temperature distribution. Therefore the interphase boundary motion can be determined by the rate of heat transfer in the system. In the recent review [12] our theory of the kink motion of the interphase boundary $[14,16,17]$ has been examined. The necessity of taking into account processes of dissipation of energy released (or additionaly absorbed) during the time of transition has been analysed [12]. The electric-field driven kinetics of ferroelectric interphase boundaries have not been studied up to now.

In this paper we obtain a criterion of necessity of considering the heat transfer effect on the interphase boundary propagation. We show here that at first-order phase transitions in ferroelectrics-semiconductors the thermal conductivity is not a controlling process and 
the interface movement is controlled by the kinetics of the phase transition. We also consider the electric field dynamics of interphase boundaries provided they are governed by the polarization evolution.

Let us find the velocity of an interphase boundary due to latent heat transfer. The heat flowing across the interphase boundary is $k \nabla T S \Delta t$, where $k$ is the thermal conductivity coefficient, $\nabla T$ is the temperature gradient, $S$ is the square of the area of the interface, $\Delta$ $t=\Delta R / v_{T}$, where $\Delta R$ is the change of size of the new phase during the interface motion with a velocity $v_{T}$. This heat per unit mass is the latent heat $L: L=k \nabla T \Delta V / \Delta m v_{T}$, $\Delta m / \Delta V$ is the density $\rho$. Thus, the propagation rate of the phase transition front due to latent heat transport is given by

$$
v_{T}=\frac{k(\nabla T)}{\rho L}
$$

Eq. (1) is the equation for the thermal balance between the heat generated by the phase transition and that conducted away. The velocity $v_{T}$ is determined by the rate at which heat released at the advancing, for example, paraelectric-ferroelectric fronts in the case of ferroelectrics.

The velocity of the interphase boundary movement caused by the kinetic process $v$ was calculated in [14]. It has been derived from the time-dependent Ginzburg-Landau equation

$$
\frac{\partial P}{\partial t}=-\Gamma \frac{\delta F(P)}{\delta P}
$$

which can be presented as follows

$$
\frac{\partial P}{\partial t}=\Gamma\left(2 D \nabla^{2} P-\frac{\partial f}{\partial P}\right)
$$

where $\Gamma$ is the Landau-Khalatnikov kinetic coefficient which is independent of temperature, $D$ is the inhomogeneity coefficient,

$$
f(P)=\frac{1}{2} A(T) P^{2}-\frac{1}{4} B P^{4}+\frac{1}{6} C P^{6}
$$

$B$ and $C$ are constants, $A=A$ ' $\left(T-T_{0}\right), T_{0}$ is the stability limit of the paraelectric phase, $A^{\prime}$ is a constant. The relation between $F$ and $f$ is given by

$$
F(P)=\int\left[D(\nabla P)^{2}+f(P)\right] d V
$$

$V$ is the volume. For a moving phase transition front Eq. (2) holds at the interphase boundary provided the interphase boundary width $\Delta$ is considerably greater than the interatomic distances. Eq. (3) cannot be solved exactly in the three-dimensional case but if the size of clusters of the new phase inside the old one $R$ is much larger than the width of the interphase boundary $\Delta: R>>\Delta$, the exact solution can be obtained [14]. Thus, the theory works provided $R>>\Delta>>d, d$ is the lattice parameter.

Relation between a critical size $R_{c}$ of the product transformed region and the growth rate $v$ may be established. We assume that the spatial dependence of the polarization $P(r)$ is approximately given by 


$$
\begin{array}{llll}
P & =P_{F} \quad \text { at } \quad|r|<R ; \\
P & =\left(\frac{P_{F}}{\sqrt{2}}\right)\left[1-\tanh \left(\frac{r-R}{2 \Delta}\right)\right]^{0.5} & \text { at } & R \leq r \leq R+\Delta ; \\
P=0 & \text { at } \quad|r| \geq R+\Delta,
\end{array}
$$

where $P_{F}^{2}=(B / 2 C)\left\{1+\left[1-\left(4 A C / B^{2}\right)\right]\right\}$. Eq. (6) gives a radial symmetric cluster of the nucleating phase embedded in a spatially uniform, metastable background. The profile for the front applied here is obtained from the exact solution of Eq. (3) in the equilibrium limit. Using (6) and integrating in (5) for $R>>\Delta$ one obtains

$$
F=-\frac{4 \pi R^{3} f\left(P_{F}\right)}{3}+\frac{\pi R^{2} D P_{F}^{2}}{\Delta}
$$

This means that the surface tension $\sigma=D P_{F}^{2} / 4 \Delta$. According to the classical theory of nucleation, the new phase nucleus whose radius corresponds to the maximum of the free energy change is the critical nucleus $R_{c}$. Then after maximization of $(7)$ we derive

$$
R_{c}=\frac{6 D}{\Delta\left|4 A-B P_{F}^{2}\right|} .
$$

Combining the interface velocity $[16]$ and (8) we obtain

$$
v=\frac{4 \Gamma D}{R_{c}}
$$

The propagation of the interphase boundary is determined by the kinetics of the order parameter, if $v_{T}>>v$. Comparing the velocity of the interphase boundary from [16] and (9) and supposing that $\nabla T \approx \frac{\Delta T}{R_{c}}(\Delta T$ is the supercooling or superheating) one obtains that this criterion is fulfilled for

$$
\frac{k \Delta T}{4 \rho L \Gamma D}>>1
$$

This condition does not depend on the critical size $R_{c}$. Let us check the criterion for ferroelectric perovskite semiconductors for which many measurements of interphase boundary dynamics have been carried out [2-12]. For $\mathrm{BaTiO}_{3}-\mathrm{PbTiO}_{3} \mathrm{~L}=8.96 \cdot 10^{6} \frac{\mathrm{erg}}{\mathrm{gr}}\left(50 \frac{\mathrm{cal}}{\mathrm{mol}}\right)$ $[21], k=11 \cdot 10^{-3} \frac{c a l}{c m \cdot s e c \cdot K}[22], \rho=5.9 \frac{g r}{c^{3}}[22], \Gamma=1.61 \cdot 10^{10} \mathrm{~Hz}[10], D=3.3 \cdot 10^{-16}$ $\mathrm{cm}^{2}[16], \Delta T=0.25 \mathrm{~K}[10], A^{6}=9.09^{\prime} \cdot 10^{-6} 1 / \mathrm{K}[16], \mathrm{B}=1.27 \cdot 10^{-12}$ esu [23], $\mathrm{C}=$ $5.73 \cdot 10^{-23}$ esu [23], $T_{c}-T_{0}=43 \mathrm{~K}[24]$. Then the required ratio is about 100 . Thus, the heat transfer process is more rapid than the kinetics of the order parameter relaxation. For this reason, the latter process determines the interface propagation in above ferroelectricssemiconductors. Consequently, the release of the latent heat of the phase transition does not play an important role in the dynamics of interphase boundaries in perovskites under consideration. However, if $\frac{k \Delta T}{4 \rho L \Gamma D} \approx 1$, the two processes must be taken into account. The theory of the kink-type motion for interphase boundaries $[14,16,17]$ is suitable for perovskite ferroelectrics-semiconductors because of fulfilling the criterion (10). The latter is caused by the comparatively high thermal conductivity and comparatively low latent heat.

At low electric fields the relaxation kinetics of polarization also dominates the thermal conduction-driven mechanism for motion of the phase transition front. For instance, in 
[25] the front velocity is $10^{-3} \frac{\mathrm{cm}}{\mathrm{sec}}$ at the electric field $E=300 \frac{\mathrm{V}}{\mathrm{cm}}$. Thus, the criterion (10) is fulfilled. The appearance of a nucleus of the new phase results in a free energy change which is given by $-\frac{4 \pi R^{3} E P_{E}}{3}+\frac{4 \pi R^{3} Q}{3}+4 \pi R^{2} \sigma$ in the linear field approximation. The first term was introduced in [26]. The second term presents the volume strain energy contribution [27]. In ferroelectric semiconductors under investigation the polarization charge at the interphase boundary can be easily compensated so that the depolarization energy is much small compared the elastic energy [3] and it may be neglected in our consideration. Then after maximisation we derive $R_{c}=\frac{2 \sigma}{P_{F}\left(E-E_{c}\right)}$, where $E_{c}=Q / P_{F}$. The latter relation reflects the fact that at first-order phase transitions the volume and shape change associated with the transition cause a strain energy contribution to the free energy supressing the nucleation process [28]. Thus, we arrived at an electrical modification of the known Laplace formule [29] in which $P_{F} E-Q$ yields the difference between the pressures in the two phases. To find the velocity of the interface motion in the presence of $E-E_{\mathrm{c}}$ in the linear field approximation, we shøuld compare the power lost by the system due to damping and the power input, i.e. the work per time unit done by the electric force. Using the dynamic form of the front profile (6) [14], calculating $\frac{\partial F}{\partial t}$, integrating and equating the two powers we obtain

$$
v=\frac{2 \Gamma D P_{F}\left(E-E_{c}\right)}{\sigma}
$$

Eq. (11) implies that the interphase boundary would move if the electric field is more than the threshold electric field $E_{c}$. This result is known from measurements of velocities of the electric field-induced motion of ferroelectric interphase boundaries in SbSI [30] and domain walls in $\mathrm{BaTiO}_{3}$ [31], Rochelle Salt [32] and $\mathrm{NaNO}_{2}$ [33]. Observations in [30] indicated the existence of some critical value of the electric field below which the boundary did not react to the presence of the electric field. The boundary began to move in fields somewhat larger than the critical value. In [31-33] $\propto \propto\left(E-E_{c}\right)$. Additional evidences in favour of the proposed theory are:

1. The kinetics observed in [6-12] are nonactivated as is seen from the temperature dependence of the front velocity [16].

2. The same value of superheating and supercooling leads to different velocities of the interphase boundary. The velocity in heating process is larger than in the cooling one [10] as it follows from the exact solution of the time-dependent Ginzburg-Landau equation [17].

3. The temperature dependence of the interface velocity is characteristic of the overdamped motion described by the relaxational kinetics of the order parameter $[16,17]$.

4. The calculated values of the front velocity coincide with experimental ones for above mentioned $\Gamma$ and $D[12,16]$. In low electric fields used, for example in [25], the criterion (10) is valid. For this the present approach is suitable.

One of us ( S.D.) is grateful to the Israel Ministry of Absorption for support. 


\section{References}

[1] Yukalov V.I., Physics Reports, 208 (1991) 395.

[2] Burfoot J.C. and Parker B.J., Brit. J. Appl. Phys. 17 (1966) 213-226.

[3] DiDomenico M., Jr. and Wemple S.H.,Phys. Rev. 155 (1967) 539-545.

[4] Surowiak Z., Dec J., Gavrilyatchenko V.G., Semenchev A.F., and Fesenko E.G., Sov. Phys. Sol. State 20 (1978) 1409-1410.

[5] Surowiak Z., Dec J., Gavrilyatchenko V.G., Semenchev A.F., and Fesenko E.G., Sov. Phys. Sol. State 20 (1978) 1411-1412.

[6] Yufatova S.M., Sindeyev Y.G., Gavrilyatchenko V.G., and Fesenko E.G., Ferroelectrics 26 (1980) 809-812.

[7] Dec J., Ferroelectrics 69 (1986) 187-200.

[8] Dec J., J. Phys. C: Solid State Phys. 21 (1988) 1257-1263.

[9] Dec J. and Kwapulinski J., Phase Transitions 18 (1989) 1-9.

[10] Dec J., Ferroelectrics bf 89 (1989) 193-200.

[11] Dec J. and Yurkevich V.E., Ferroelectrics 110 (1990) 77-83.

[12] Dec J., Phase Transitions 45 (1993) 35-52.

[13] Metiu H., Kitahara K., and Ross J., J. Chem. Phys. 64 (1976) 292-297.

[14] Dorfman S., Fuks D., and Gordon A., Phys. Rev. B52 (1995) 12473-12476.

[15] Soboleva T.K., Stepanovskii E.P., and Sukstanskii A. L., JETP Lett. 42 (1985) 68-69.

[16] Dorfman S., Fuks D., Gordon A., Postnikov A.V., and Borstel G., Phys. Rev. B52 (1995) 7135-7141.

[17] Gordon A., Dorfman S., and Wyder P., Phys. Rev. B52 (1995) 143-148.

[18] Tuszyńskii J.A., Otwinovski M., and Dixon J.M., Phys. Rev. B44 (1991) 9201-9213.

[19] Fillipov A.E., Kuzovlev Yu.E., and Soboleva T.K., Phys. Lett. A178 (1993) 301-309.

[20] Mamin R.F., JETP Lett. 60 (1994) 52-54.

[21] Shirane G. and Takeda A., J. Phys. Soc. Japan 7 (1952) 1-12.

[22] Glower D.D. and Wallace D.C., J. Phys. Soc. Japan 18 (1963) 679-684.

[23] Ramirez R., Lapeña M.F., and Gonsalo J.A.; Phys. Rev. B42 (1990) 2604-2606.

[24] Samara G.A., Ferroelectrics 2 (1971) 277-289.

[25] Fridkin V.M., Gorelov I.M., Grekov A.A., Lyakhovitskaya V.A., and Rodin A.I., Sov. Phys. JETP Lett. 4 (1966) 310-311.

[26] Langer J.S., Annals of Physics 41 (1967) 108-157. 
[27] Ingle S.G. and Joshi S.C., Phys. Rev. B34 (1986) 4840-4845.

[28] Cook H.E., Phys. Rev. B15 (1977) 1477-1488.

[29] Lifshitz E.M. and Pitaevskii L.P., Statistical Physics. Part I, (Pergamon Press, Oxford, 1980).

[30] Travina T.S., Golik L.L., Elinson M.I., Ignatkin V.I., and Lyakhovitskaya V.A., Sov. Phys. Sol. State 11 (1969) 500-505.

[31] Merz W.J., Phys. Rev. 95 (1954) 690-698.

[32] Mitsui T. and Furuichi J., Phys. Rev. 95 (1954) 558.

[33] Hatta I. and Sawada S., Japan J. Appl. Phys. 4 (1965) 389-393. 